\title{
Almacenamiento de carbono y caracterización lignocelulósica de plantaciones comerciales de Tectona grandis L.f. en México
}

\author{
Carbon storage and lignocellulosic characterization of commercial plantations \\ of Tectona grandis L.f. in Mexico
}

\author{
Bayron Alexander Ruiz Blandon ${ }^{\circledR}$, Efrén Hernández Álvarez ${ }^{\circledR}{ }^{\circledR}$ Eduardo Salcedo Pérez $^{(}{ }^{\circledR}$, \\ Ramón Rodríguez Macías ${ }^{4^{\circledR}}$, Agustín Gallegos Rodríguez $z^{{ }^{\circledR}}$, Eduardo Valdés Velarde ${ }^{6^{(}}$ \\ y Rufo Sánchez Hernández ${ }^{7}$
}

Ruiz-Blandon, B.A., Hernández-Álvarez, E., Salcedo-Pérez, E., Rodríguez-Macías, R., Gallegos-Rodríguez, A., Valdés-Velarde, E. y Sánchez-Hernández, R. (2019). Almacenamiento de carbono y caracterización lignocelulósica de plantaciones comerciales de Tectona grandis L.f. en México. Colombia Forestal, 22(2), 15-29.

Recepción: 25 de septiembre de 2018

\section{Resumen}

La teca es una planta exótica introducida en México por el valor de su madera. El objetivo del estudio fue calcular el carbono almacenado en la biomasa vegetal y suelo para luego determinar la composición lignocelulósica de la madera en plantaciones de teca a los seis años en el estado de Nayarit, México. En tres plantaciones se colectó información dendrométrica y biomasa de 75 árboles, también de mantillo y suelo para determinar sus propiedades. Las diferencias fueron significativas $(p \leq 0.0001)$ al comparar la plantación de mayor y menor crecimiento en DAP (54\%), altura (51.3\%) y área basal (78.7\%) siendo los suelos de mejor $\mathrm{pH}$ (6.2); así como el rendimiento en biomasa aérea $(59.4 \%)$, carbono en la teca $(60.2 \%)$, mantillo (30.3 \%) y suelo $(41.9 \%)$ por superficie, sin diferencias en los contenidos lignocelulares. Se recomienda estudiar las raíces y nutrientes
Aprobación: 4 de junio de 2019

del suelo pues condicionan la eficiencia de la teca en torno al cambio climático.

Palabras clave: biomasa, celulosa, DAP, mantillo, método directo, modelos alométricos, propiedades del suelo.

\begin{abstract}
Teak is an exotic plant introduced to Mexico for its wood value. The aim of this study was to quantify the carbon stored in the plant biomass and soil and to determine the wood lignocellulosic composition in 6-years-old teak plantations in the Nayarit state, Mexico. In three plantations, dendrometric and biomass information were collected from 75 trees, as well as litter layer and soil to determine their properties. The differences were significant $(p \leq 0.0001)$ when comparing the plantation with the highest and lowest growth in DBH (54\%), height (51.3\%) and
\end{abstract}

\footnotetext{
Departamento de Botánica y Zoología. Centro Universitario de Ciencias Biológicas y Agropecuarias (CUCBA), Universidad de Guadalajara (UdeG). Zapopan, Jalisco, México. Correo electrónico: toxbombaso@yahoo.es. Autor para correspondencia.

Departamento de Producción Forestal. CUCBA, UdeG. Zapopan, Jalisco, México. Correo electrónico: efren.hernandez@academicos.udg.mx. Departamento de Botánica y Zoología, CUCBA. Zapopan, Jalisco, México. Correo electrónico: eduardo.salcedo@academicos.udg.mx. Departamento de Botánica y Zoología, CUCBA, UdeG. Zapopan, Jalisco, México. Correo electrónico: ramonrod@cucba.udg.mx.

Departamento de Producción Forestal. CUCBA, UdeG. Zapopan, Jalisco, México. Correo electrónico: agustin.gallegos@academicos.udg.mx. Departamento de Fitotecnia. Universidad Autónoma Chapingo (UACh). Texcoco, México, México. Correo electrónico: velarde@correo. chapingo.mx.

División Académica de Ciencias Biológicas, Universidad Juárez Autónoma de Tabasco (UJAT). Villahermosa, Tabasco, México. Correo electrónico: rufo.sanchez@ujat.mx.
} 
basal area $(78.7 \%)$ being the soils with the best $\mathrm{pH}$ (6.2); as well as, the aerial biomass yield (59.4\%), teak carbon $(60.2 \%)$, litter layer $(30.3 \%)$ and soil $(41.9 \%)$ per surface, without differences in lignoceIlular contents. It is recommended to study the roots and soil nutrients, since they condition the teak efficiency regarding the climate change.

Key words: biomass, cellulose, DBH, litter layer, direct method, allometric models, soil properties.

\section{INTRODUCCIÓN}

La teca (Tectona grandis L.f.) es un árbol de rápido crecimiento perteneciente a la familia Lamiaceae; es considerada una de las especies maderables más conocidas en el mundo debido a la calidad de la madera. Lo anterior, le confiere gran importancia comercial por lo que esta se ha extendido en más de 70 países, aproximadamente, siendo los principales productores India, Indonesia, Myanmar y Tailandia (Zhou et al., 2012; Sreejesh et al., 2013; GRIN, 2018).

Para México, la teca es una planta exótica que actualmente se encuentra en auge, lo cual ha despertado interés por aumentar sus extensiones en el país. Sin embargo, la superficie plantada con especies latifoliadas en México ascendió a 149 959, en la que la teca representó aproximadamente el $12 \%$ (18 009 ha), ocupando el quinto lugar de importancia con tendencias al crecimiento y siendo los estados referentes Chiapas, Campeche, Quintana Roo, Veracruz, Tabasco y Nayarit (Conafor, 2013). Además de las bondades socioeconómicas de la teca, también se ha estudiado su potencial en torno al cambio climático, pues tiene efectos negativos en los procesos ecológicos, económicos y sociales que interactúan en la tierra.

El cambio climático es producido principalmente por los gases de efecto invernadero (GEI), siendo el más impactante el $\mathrm{CO}_{2}$ por los volúmenes producidos anualmente (Ibrahim et al., 2007; Parra et al., 2013; FAO, 2017). El $\mathrm{CO}_{2}$ es causado directamente por las acciones humanas que modifican el medioambiente natural, destacándose la deforestación, cambio del uso del suelo y la quema de combustibles fósiles (Ibrahim et al., 2007; González y Gómez, 2015). El CO $\mathrm{CO}_{2}$ ha incrementado su concentración atmosférica en los últimos 60 años, pues ha pasado de 280 a 400 ppm. México es de los países más contaminantes y aporta el $2 \%$ de los GEl en el mundo (Ordóñez y Masera, 2001; IPCC, 2007; Grünewald et al., 2014).

Una de las alternativas propuestas para mitigar el cambio climático acelerado es el establecimiento de plantaciones con especies forestales de rápido crecimiento, puesto tienen la capacidad de captar el $\mathrm{CO}_{2}$ atmosférico y fijar el $\mathrm{C}$ en el suelo dado que los árboles pueden transforman alrededor de 120 GtC en biomasa y el suelo almacenar $2300 \mathrm{GtC}$ (IPCC 2007; Almeida et al., 2010). Estudios previos relacionados con la estimación de biomasa y $\mathrm{C}$ en plantaciones de teca a la edad de cinco años en la India demostraron que la ganancia en biomasa seca fue de $67.81 \mathrm{~kg}$.árbol $^{-1}$ siendo equivalentes a $29.5 \mathrm{kgC}^{2}$ árbol ${ }^{-1}$, lo cual aumenta paulatinamente con la edad ( $p$. ej. 50 años $=1052.2$ kg.árbol $^{-1}$ y $464.95 \mathrm{kgC.árbol}^{-1}$ [Sreejesh et al., 2013]). Lo anterior invita a la búsqueda de opciones que permitan calcular con precisión el C almacenado en plantaciones forestales, teniendo en cuenta factores hidroclimatológicos y edáficos. Es por todo lo anterior que la presente investigación tiene importancia. Adicionalmente, el C capturado por los árboles a través de la fotosíntesis es transformado principalmente en biomasa compuesta químicamente por sus componentes lignocelulares (Pettersen, 1984; Windeisen et al., 2003) pese a lo antes mencionado, la eficiencia de las especies forestales en la captura de $\mathrm{C}$ dependerá de su adaptabilidad en áreas exóticas y el manejo silvícola. 
Por lo tanto, esta investigación planteó como hipótesis que las plantaciones comerciales de teca (PCT) en sus primeras etapas de crecimiento condicionan su eficiencia en la formación de biomasa y acumulación de $C$ en sus principales componentes según propiedades básicas del suelo, sin afectar significativamente la composición lignocelulósica de la madera. Los objetivos de la presente investigación fueron: 1) cuantificar el $\mathrm{C}$ almacenado en la biomasa total aérea de la teca (BTA), mantillo y suelo a partir de mediciones directas en campo; 2) determinar la composición lignocelulósica de la madera en tres plantaciones comerciales de seis años en el estado de Nayarit, México. La medición directa de la biomasa de la teca, permitió la construcción de modelos alométricos comprobados por procedimientos estadísticos, los cuales facilitarán el inventario de biomasa en futuras investigaciones forestales.

\section{MATERIALES Y MÉTODOS}

\section{Área de estudio}

Las plantaciones de teca Cerritos 1 (PCTC1), Cerritos 2 (PCTC2) y El Mirador 2 (PCTM2) se ubican en el municipio de Ruiz, estado de Nayarit, México, localizadas entre los 39 y $41 \mathrm{~m}$ de altitud, a los $21^{\circ} 54^{\prime} 10^{\prime \prime}$ latitud norte-105'03'29" longitud oeste; $21^{\circ} 54^{\prime} 00^{\prime \prime}$ latitud norte- $105^{\circ} 03^{\prime} 27^{\prime \prime}$ longi-

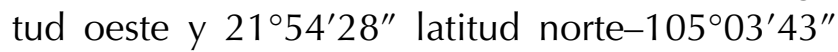
longitud oeste, respectivamente. Las PCT en propiedad de la empresa Agroforestal Nayarita S. A. se establecieron en junio de 2008 bajo condiciones hidroclimáticas iguales (figura 1). Según la clasificación de Köppen modificada por García (1987), el área de influencia de las PCT se caracteriza por tener un ambiente cálido subhúmedo con lluvias en verano $\left(A_{(w)}\right)$, precipitación

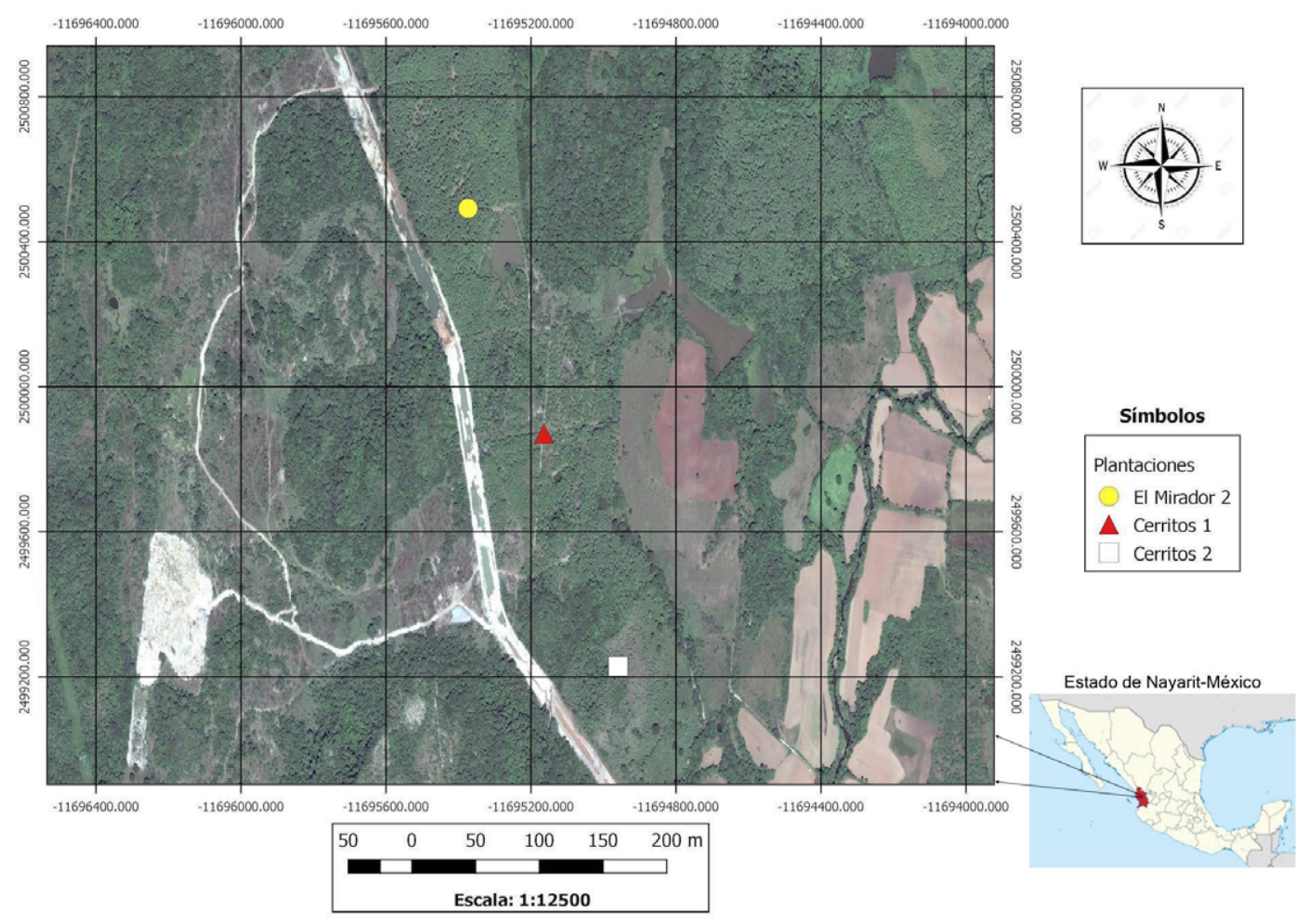

Figura 1. Geolocalización de las plantaciones comerciales de teca (PCT) en el municipio de Ruiz, estado de Nayarit, México. 
anual de $1496.7 \mathrm{~mm}$, siendo enero, febrero, marzo, abril, mayo, noviembre y diciembre los meses más secos (Inegi, 2017). La temperatura media anual promedio es de $23.5^{\circ} \mathrm{C}$ destacándose julio como el mes más caluroso, sin embargo, geológicamente los suelos son originarios de rocas ígneas extrusiva de dominancia Cambisoles Epiléptico (cm lep), mayormente cubiertos por selva caducifolia (Inegi, 2017).

Antes de establecer las PCT se removió la vegetación mediante la aplicación de técnicas silvícola, lo cual permitió el aprovechamiento de las especies encino prieto (Quercus emoryi), tepeguaje (Lysiloma acapulcensis (Kunth), papelillo (Bursera odorata), guamúchil (Pithecellobium dulce [Roxb.] Benth.), Huizache (Acacia pennatula [Schltdl. \& Cham.] Benth.) y guanacaste (Enterolobium cyclocarpum [Jacq.] Griseb). Posteriormente, el excedente de cobertura vegetal fue quemada de manera controlada. A los sitios previamente tratados, se les hizo análisis físicos y químicos del suelo, estos presentaron textura franca (arcillosa o arenosa dependiendo el sitio), $\mathrm{pH}$ promedio $<5.3, \mathrm{MO}<0.5 \%$, fueron bajos en concentración de $\mathrm{N}\left(<200 \mathrm{mg} \cdot \mathrm{kg}^{-1}\right), \mathrm{P}$ disponible $\left(10 \mathrm{mg} \cdot \mathrm{kg}^{-1}\right), \mathrm{K}$ (<3 mg. $\left.\mathrm{kg}^{-1}\right), M n\left(3 \mathrm{mg} \cdot \mathrm{kg}^{-1}\right), \mathrm{Mg}\left(170 \mathrm{mg} \cdot \mathrm{kg}^{-1}\right)$ y $\mathrm{Cu}\left(0.4 \mathrm{mg} \cdot \mathrm{kg}^{-1}\right)$; medianamente alto en $\mathrm{Ca}$ intercambiable (67\%), así como elevados en hierro Fe (>600 mg. $\left.\mathrm{kg}^{-1}\right)$. Debido a lo anterior, se aplicó fertilizante con formulación 50-120-75 (NPK), 3000 kg.ha-1 de hidróxido de Ca, 250 kg.ha-1 de fosfato diamónico, $63 \mathrm{~kg}$.ha-1 de cloruro de potasio y $75 \mathrm{~kg} \cdot \mathrm{ha}^{-1}$ de sulfato de potasio con el fin de mejorar su calidad.

Las semillas fueron suministradas por la Comisión Nacional Forestal (Conafor) provenientes del Banco de Semillas Forestales (BSF) del Centro Agronómico Tropical de Investigación y Enseñanza (Catie) Costa Rica; las semillas presentaron un 99 $\%$ de pureza y $61 \%$ de germinación. Cuando las plántulas de teca alcanzaron los 8 meses de edad en vivero $(20 \mathrm{~cm}$ de altura y $8 \mathrm{~mm}$ de diámetro), fueron trasplantadas al suelo bajo el sistema de marco real en disposición espacial de 3.5 × 3.5 m (918 plantas.ha-1). El manejo de las plantaciones fue uniforme, basado en podas de formación, control de arvenses, plagas, aclareos del $50 \%$ una vez realizado el muestreo (6 años) y riegos de emergencia en las épocas de sequía.

\section{Dendrometría}

Se establecieron tres parcelas de $7 \times 25.4 \mathrm{~m}$ (alrededor de $533.6 \mathrm{~m}^{2}$ ) distribuidas en bloques completamente al azar en cada PCT. Las variables medidas en 75 árboles por plantación fueron el diámetro a la altura del pecho (DAP) tomado a $1.30 \mathrm{~m}$ con cinta diamétrica y la altura total $(\mathrm{H}) \mathrm{a}$ una distancia de 15 m con el hipsómetro Haga; además, el área basal (AB) se calculó según Cancino (2006). Las mediciones se realizaron en junio de 2014.

\section{Muestreo de la biomasa}

Se derribaron 75 árboles en cada PCT donde la biomasa fue seccionada por componente (hojas, ramas y fuste) y pasada en una báscula digital de plataforma con capacidad de $100 \mathrm{~kg} \pm 10 \mathrm{~g}$; esto permitió determinar su peso en fresco (psb) según Parada et al. (2010). La cuantificación de la biomasa se llevó a cabo en junio de 2014.

\section{Muestreo del mantillo y suelo}

Tomando como referencia cuatro árboles, se colectaron muestras de mantillo en $1 \mathrm{~m}^{2}(n=6)$. Posteriormente, se pesaron en una báscula digital de plataforma con capacidad de $40 \mathrm{~kg} \pm 2 \mathrm{~g}$, permitiendo la determinación de su peso en fresco (Schlegel, 2001). Alrededor del $80 \%$ del mantiIlo correspondió a hojas, $15 \%$ en ramas y el $5 \%$ en otros materiales de origen orgánico necrosados. En los mismos puntos de muestreo del mantillo se tomaron muestras de suelo $(n=6)$ a $30 \mathrm{~cm}$ de profundidad con el fin de precisar sus propiedades en laboratorio. El muestreo del mantillo y suelo fue realizado en junio de 2014. 


\section{Análisis físico y químico del suelo}

Las propiedades de los suelos se determinaron en el Laboratorio de Suelos y Agua del Centro Universitario de Ciencias Biológicas y Agropecuarias (CUCBA) de la Universidad de Guadalajara (UdeG) aplicando la norma NOM-021-RECNAT-2000 (DOF, 2002), además, se hicieron seis repeticiones por experimento. La textura fue determinada con el hidrómetro de Bouyoucos, densidad aparente (Da) por el método del terrón, el pH en agua (1:2) con un potenciómetro Orion Star A210, la capacidad de intercambio catiónico $(\mathrm{CIC})$ en solución de acetato de amonio, la concentración de $\mathrm{C}$ orgánico $(\mathrm{C} \%)$ en el analizador Shimadzu TOC 5050-A y este fue convertido a materia orgánica $(\mathrm{MO})$ multiplicado el porcentaje de $\mathrm{C}$ promedio en la $\mathrm{MO}(58 \%)$, respectivamente.

\section{Concentración de carbono en la biomasa vegetal, mantillo y suelo}

Las muestras de biomasa vegetal fueron secadas en horno a $70 \pm 3{ }^{\circ} \mathrm{C}$ hasta alcanzar un peso constante. Luego, fueron molidas y tamizadas en malla 60. A partir de $2 \mathrm{mg}$ de muestra se cuantificó la concentración de $\mathrm{C}$ total por combustión, usando el analizador elemental CHSN-O Leco TruSpec $\mathbb{R}$ Micro $(n=75)$. Para el caso del suelo, a partir de 1 g de muestra previamente secado al ambiente y tamizado (maya 60) se calculó la concentración de C orgánico por combustión usando el analizador TOC Shimadzu 5050-A ( $n=6)$.

\section{Biomasa, modelos alométricos y carbono en la teca}

Una vez determinado el pfb de la teca se tomó un $1 \mathrm{~kg}$ de hojas, ramas y fuste; posteriormente, fue secada en horno a $100 \pm 3{ }^{\circ} \mathrm{C}$ hasta alcanzar un peso constante con el propósito de determinar su peso seco $(p s b)$. La siguiente fórmula se usó para calcular la biomasa seca (BS):

$$
B S=p f b-p s b
$$

Donde, $B S$ es biomasa seca $(\mathrm{kg})$, pfb el peso fresco de la biomasa $(\mathrm{kg})$ y psb el peso seco de la biomasa (kg) (Parada et al., 2010).

Los modelos alométricos fueron construidos aplicando los principios descritos por Segura y Andrade (2008) y Parada et al. (2010). Calculada la biomasa seca por componente $(n=27)$, se hizo un análisis de correlación de Pearson con el fin de identificar la variable más importante (DAP, H y $A B)$ para estimar la biomasa en hojas ramas, fuste $y$ BAT. Tomando el DAP, la $R^{2} \leq 0.7$ en hojas, $R^{2} \leq 0.6$ en ramas, $R^{2} \leq 0.9$ en fuste y $R^{2} \leq 0.9$ en BTA, sin correlación con la $\mathrm{H}$; el $\mathrm{AB}$ sin correlación para hojas y ramas $y, R^{2} \leq 0.7$ en fuste y $B T A$. Lo anterior sugirió la elaboración de las ecuaciones a partir del DAP mediante el análisis de regresión simple, siendo el modelo ideal aquel que $R^{2} \leq 1$ y el error estándar (Ee) menor a 1. Los análisis de regresión múltiple generaron modelos con $\mathrm{R}^{2} \leq 0.7$ y el error estándar (Ee) por encima de 10. La conversión de biomasa a C se hizo multiplicando la biomasa seca por componentes por la concentración de $\mathrm{C}$ determinada instrumentalmente (Parada et al., 2010).

\section{Carbono en mantillo}

Se tomaron $200 \mathrm{mg}$ de muestra $(n=6)$, la cual fue secada en un horno a $100 \pm 3{ }^{\circ} \mathrm{C}$, hasta alcanzar un peso constante. Mediante la siguiente fórmula se determinó el contenido de humedad:

$$
C H=\frac{P h s-P s s}{P s s} \times 100
$$

Donde, $\mathrm{CH}$ es el contenido de humedad (\%), Phs es el peso húmedo de la muestra (g) y PsS es el peso seco de la muestra (g). Conociendo el $\mathrm{CH}$, se calculó la proporción del peso húmedo que correspondió a la biomasa total mediante la siguiente fórmula:

$$
B=\frac{P h B t}{1} \times\left(\frac{C H}{100}\right)
$$

Donde, $B$ es la biomasa seca en (g), PhBt es el peso húmedo total de biomasa (g), $\mathrm{CH}$ es la humedad (\%) (Schlegel et al., 2001). 


\section{Carbono en el suelo (COS)}

Para calcular el C almacenado en el suelo se empleó la siguiente fórmula:

$$
C O S=D a \times \operatorname{Pr} \times C
$$

Donde, COS es el C orgánico del suelo $\left(\mathrm{Mg}_{\mathrm{h}} \mathrm{ha}^{-1}\right)$, Da es la densidad aparente en $\left(\mathrm{g} \cdot \mathrm{Cm}^{-3}\right), \operatorname{Pr}$ indica la profundidad del suelo $(\mathrm{cm})$ y $C$ es el $C$ en porcentaje (González-Molina et al., 2008).

\section{Caracterización lignocelulósica de la madera de la teca}

En las plantaciones evaluadas fueron seleccionados tres árboles. De estos se extrajeron cilindros de $40 \mathrm{~cm}$ de largo y tomada en el área del DAP, luego se cortaron en discos de $4 \mathrm{~cm}$, se descortezaron, astillaron, secaron en un horno a $70 \pm 3{ }^{\circ} \mathrm{C}$, hasta alcanzar un peso constante, se molieron y tamizaron (maya 60). Para la determinación de la composición lignocelulósica de la madera se usaron las normas Tappi (The Technical Association of Pulp and Paper Industry) ( $n=6)$. La madera fue liberada de extraíbles según Tappi Standards Test 264 cm-07 (Tappi, 2007).

\section{Contenido de extraíble (Tappi Standards Test 204 cm-07)}

En 2 g de muestra $(n=6)$ ser realizaron extracciones con acetona y etanol al $95 \%$; además de diclorometano al $98 \%$ en equipo Soxhlet por $5 \mathrm{~h}$. La muestra se secó en un horno a $105 \pm 3{ }^{\circ} \mathrm{C}$ por $1 \mathrm{~h}$ y el porcentaje de extraíbles se calculó mediante la siguiente fórmula:

$$
E=\left[\left(\frac{P e-P r b}{P m}\right)\right] \times 100
$$

Donde, $E$ indica extraíbles (\%), Pe es el peso del extraíble (g), Prb es el peso del residuo del blanco (g) y Pm el peso de la muestra (g) (Tappi, 2007).

\section{Contenido de cenizas (Tappi Standards Test 211 om-12)}

Se tomaron $2 \mathrm{~g}$ de muestra $(n=6)$ e introdujeron en una mufla a $525 \pm 5{ }^{\circ} \mathrm{C}$ por $6 \mathrm{~h}$. El cálculo de las cenizas presentes en la muestra se realizó mediante la fórmula:

$$
C=\frac{A}{B} \times 100
$$

Donde, $C=$ ceniza $(\%), A$ es el peso de la ceniza (g) y $B$ el peso de la muestra base seca (g) (Tappi, 2012).

\section{Contenido de ligninas Klason (Tappi Standards Test 222 om-11)}

Se tomó 1 g de muestra ( $n=6)$, se mezcló con ácido sulfúrico al $72 \%$ manteniéndose en agitación y baño maría $\left(20 \pm 3{ }^{\circ} \mathrm{C}\right)$ por 2 h; se diluyó la solución hasta alcanzar una concentración de ácido del $3 \%$ y un volumen de $575 \mathrm{ml}$. Posteriormente, se hirvió en un sistema de reflujo por 4 h, se filtró a través de un crisol tipo Gooch, se lavó con agua caliente y fue secado a $105 \pm 3{ }^{\circ} \mathrm{C}$, hasta alcanzar un peso constante. Las ligninas presentes en las muestras se cuantificaron según la siguiente fórmula:

$$
L=\frac{(P l \times 100)}{P m l e}
$$

Donde, $L$ es la lignina (\%), $P l$. el peso de la lignina (g) y Pmle el peso de la muestra libre de extraíbles (g) (Tappi, 2011).

\section{Contenido de holocelulosas}

En $5 \mathrm{~g}$ de muestra $(n=6)$ fueron agregados $1.5 \mathrm{~g}$ de clorito de sodio, se transfirió a un matraz con $100 \mathrm{ml}$ de agua y se adicionaron 10 gotas de ácido acético. El matraz con la disolución se puso en agitación constante en baño maría $\left(80{ }^{\circ} \mathrm{C}\right)$ y se añadieron $1.5 \mathrm{~g}$ de clorito de sodio y 10 gotas de ácido acético cada 60 min durante 4 h. Los 
matraces se dejaron enfriar a temperatura ambiente y la muestra se filtró en vacío (Wise et al., 1946).

\section{Contenido de hemicelulosas}

Se estimaron basados en la diferencia entre $100 \%$ y la suma del porcentaje de celulosa y lignina en la madera libre de extraíbles(n=6) (Núñez, 2008).

\section{Contenido de $\alpha$-Celulosa por la técnica Cross y Bevan (Tappi Standards Test 9 M)}

El porcentaje de $\alpha$-Celulosa se determinó como el procedimiento de la lignina a partir de holocelulosas $(n=6)$ (Tappi, 1954).

\section{Análisis estadístico de los datos}

Las diferencias en crecimiento, biomasa, C, propiedades del suelo y caracterización lignocelulósica de la madera se analizaron con un Anova. La normalidad de los datos fue comprobada mediante gráficos P-P Plot, por lo que tuvieron una distribución normal. La prueba de comparación de medias mínimas Tukey se usó para identificar las diferencias significativas $(p \leq 0.05)$ de las medias en relación con las PCT estudiadas. Los análisis de regresión simple permitieron la generación de modelos basados en la biomasa de la teca. El paquete estadístico usado en los anteriores procedimientos fue el SAS ${ }^{\circledR}$ v9.0 (SAS Institute, 2009).

\section{RESULTADOS}

\section{Comportamiento dendrométrico y propiedades del suelo}

A pesar de que las PCT fueron manejadas de igual forma y establecidas en condiciones hidroclimáticas semejantes, hubo diferencias en el crecimiento destacándose la PCTM2 donde los árboles presentaron mayor $\mathrm{DAP}\left(\mathrm{F}_{75,2}=258.7, \mathrm{p} \leq 0.0001\right), \mathrm{H}\left(\mathrm{F}_{75,2}=\right.$ $197.2, \mathrm{p} \leq 0.0001)$ y $A B\left(\mathrm{~F}_{75,2}=238.9, \mathrm{p} \leq 0.0001\right)$ por superficie en comparación a las PCTC2 (14.4, 13.7 y $21.3 \%$, respectivamente) y PCTC1 $(54,51.3$ y $78.7 \%$, respectivamente) siendo esta última de menor crecimiento en el área evaluada (tabla 1). En torno a las propiedades del suelo, las PCTM2 y C2 presentaron textura franco-arcillosa y la PCTC1 franco-arenosa, no obstante, la Da fue estadísticamente similar en las tres plantaciones $\left(\mathrm{F}_{6,2}=0.3\right.$, $\mathrm{p}=0.7$ ). Los suelos de la PCTM2 se caracterizaron por tener un $\mathrm{pH}$ más cercano a la neutralidad $\left(\mathrm{F}_{6,2}=5.6, \mathrm{p}=0.02\right)$, mayor concentración de $\mathrm{C}$ y $\mathrm{MO}$ $\left(\mathrm{F}_{6,2}=5.2, \mathrm{p}=0.02\right)$. La CIC fue superior en los suelos de la PCTC2 $\left(\mathrm{F}_{6,2}=1219.2, \mathrm{p} \leq 0.0001\right)$ (tabla 2$)$.

Tabla 1. Comportamiento dendrométrico promedio en plantaciones comerciales de teca (PCT) de 6 años en el estado de Nayarit, México $(n=75)$.

\begin{tabular}{lccc}
\hline Plantación & $\begin{array}{c}\text { Diámetro } \\
(\mathbf{c m})\end{array}$ & $\begin{array}{c}\text { Altura } \\
(\mathbf{m})\end{array}$ & $\begin{array}{c}\text { Área basal } \\
\left(\mathbf{m}^{\mathbf{2}} \mathbf{. h a}\right.\end{array}$ \\
\hline El Mirador 2 & $13.9 \pm 1.3$ & $11.7 \pm 1.5$ & $14.1 \pm 3.2$ \\
Cerritos 2 & $11.9 \pm 1.8$ & $10.1 \pm 1.1$ & $11.1 \pm 2.2$ \\
Cerritos 1 & $6.4 \pm 1.3$ & $5.7 \pm 1.5$ & $3.0 \pm 0.9$ \\
\hline
\end{tabular}

Valores de medias ( \pm error estándar) sin letras en cada columna son estadísticamente diferentes $(\mathrm{p} \leq 0.05)$.

Tabla 2. Propiedades del suelo en las plantaciones comerciales de teca (PCT) de 6 años en el estado de Nayarit, México $(n=6)$.

\begin{tabular}{|c|c|c|c|}
\hline \multirow{2}{*}{ Propiedad } & \multicolumn{3}{|c|}{ Plantación } \\
\hline & El Mirador 2 & Cerritos 2 & Cerritos 1 \\
\hline Profundidad $(\mathrm{cm})$ & $0-30$ & $0-30$ & $0-30$ \\
\hline Textura & $\mathrm{Cr}$ & $\mathrm{Cr}$ & $\mathrm{Ca}$ \\
\hline $\mathrm{Da}\left(\mathrm{g} \cdot \mathrm{cm}^{-3}\right)$ & $1.1 \pm 0.0^{\mathrm{a}}$ & $1.0 \pm 0.0^{\mathrm{a}}$ & $1.0 \pm 0.0^{\mathrm{a}}$ \\
\hline $\mathrm{pH}$ & $6.2 \pm 0.1$ & $5.7 \pm 0.1$ & $5.8 \pm 0.2^{\text {ba }}$ \\
\hline $\begin{array}{l}\mathrm{ClC} \\
\left(\mathrm{cmol}^{(+)} \mathrm{kg}^{-1}\right)\end{array}$ & $37.4 \pm 2.0^{\mathrm{a}}$ & $38.5 \pm 4.1^{\mathrm{a}}$ & $28.4 \pm 1.9$ \\
\hline $\mathrm{C}(\%)$ & $1.3 \pm 0.1$ & $0.7 \pm 0.1$ & $0.9 \pm 0.2^{\text {ba }}$ \\
\hline $\mathrm{MO}(\%)$ & $2.2 \pm 0.2$ & $1.2 \pm 0.2$ & $1.6 \pm 0.4^{\mathrm{ba}}$ \\
\hline
\end{tabular}

Cr: franco arcilloso. Ca: franco arenoso. Da: densidad aparente. CIC: capacidad de intercambio catiónico. C: carbono. MO: materia orgánica.

Valores de medias ( \pm error estándar) en cada fila sin letras son estadísticamente diferentes $(\mathrm{p} \leq 0.05)$. 


\section{Modelos alométricos, biomasa y carbono}

Las diferencias altamente significativas del diámetro entre las tres plantaciones permitieron generar modelos alométricos para la estimación de la biomasa hojas, ramas, fuste y BTA con valores de $\mathrm{R}^{2}$ $\geq 0.9$ y error estándar $\leq 1$, lo cual se muestra en la tabla 3. De manera interesante, la PCM2 tuvo el mayor rendimiento en BTA por árbol $\left(\mathrm{F}_{75,2}=536.5\right.$, $\mathrm{p} \leq 0.0001$ ), siendo 16.3 y $59.4 \%$ superior que las PCTC2 y C1 respectivamente (tabla 4). La distribución de la BTA por componente en la plantación de mejor rendimiento correspondió a $9.7 \%$ en hojas, $18.7 \%$ en ramas y $71.5 \%$ en el fuste. Sin embargo, dicha distribución sufrió cambios sustanciales en relación con el crecimiento, puesto que la BTA en la PCTC1 comprendió el $11.2 \%$ en hojas, 21.6 $\%$ en ramas y $67.2 \%$ en el fuste. La diferencia de BTA $\left(F_{75,2}=1237.0, p \leq 0.0001\right)$ por superficie entre

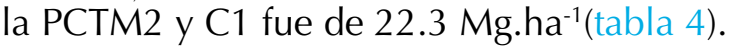

Cabe mencionar que el rendimiento de biomasa entre las plantaciones fue equivalente al $\mathrm{C}$ acumulado por árbol, por consiguiente, la ganancia de $\mathrm{C}$ $\left(\mathrm{F}_{75,2}=238.9, \mathrm{p} \leq 0.0001\right)$ en la PCTM2 fue significativamente mayor que la PCTC2 y C1 (16.6 y $59.8 \%$ ) (tabla 4). La distribución del C por componente se comportó similar a la biomasa ( $p$. ej. PCTM2= $9.2 \%$ en hojas, $\mathrm{F}_{75.2}=255.2$, $\mathrm{p} \leq 0.0001 ; 18.4 \%$ en ramas, $F_{75,2}=269.3, p \leq 0.0001 ; y 72.4 \%$ en fuste, $\mathrm{F}_{75,2}=252.1, \mathrm{p} \leq 0.0001 ;$ ). La diferencia del $\mathrm{C}$ acumulado en la BTA entre la plantación de mayor y

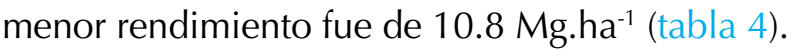

Tabla 3. Modelos alométricos para la estimación de la biomasa aérea en teca (BAT) ( $n=27)$.

\begin{tabular}{lccc}
\hline \multicolumn{1}{c}{ Componente } & Modelos alométricos & $\mathbf{R}^{2}$ & Ee \\
\hline Hojas & $B h=0.536046 * D A P$ & 0.9922 & $0.1748^{*}$ \\
Ramas & $B r=0.742697 * D A P$ & 0.9913 & $0.2561^{*}$ \\
Fuste & $B f=\exp (1.28236 * \ln (D A P))$ & 0.9963 & $0.2045^{*}$ \\
Biomasa total aérea & $B t a=\exp (1.4198 * \ln (D A P))$ & 0.9971 & $0.1994^{*}$ \\
\hline
\end{tabular}

B: biomasa seca. DAP: diámetro a la altura del pecho $(\mathrm{cm})$. Ee: error estándar. * $\mathrm{p} \leq 0.05$.

Tabla 4. Biomasa y carbono acumulado en la biomasa aérea de plantaciones de teca a los 6 años en el estado de Nayarit, México $(n=75)$.

\begin{tabular}{|c|c|c|c|c|c|c|c|c|}
\hline Plantación & Componente & $\begin{array}{c}\text { B } \\
\left(\mathrm{kg}^{-a ́ r b o l}{ }^{-1}\right) \\
\end{array}$ & $\begin{array}{c}\mathrm{C} \\
(\%)\end{array}$ & $\begin{array}{c}\mathrm{C} \\
\left(\mathrm{kg}^{-a ́ r b o l}{ }^{-1}\right) \\
\end{array}$ & $\begin{array}{c}\text { B } \\
\left(\mathrm{kg}^{\left.-h^{-1}\right)}\right)\end{array}$ & $\begin{array}{c}\mathrm{C} \\
\left(\mathbf{k g} \cdot \mathrm{ha}^{-1}\right)\end{array}$ & $\begin{array}{c}\text { B } \\
\left(\text { Mg.ha- }^{-1}\right) \\
\end{array}$ & $\begin{array}{c}\mathrm{C} \\
\left(\mathrm{Mg}^{-h^{-1}}\right)\end{array}$ \\
\hline \multirow[t]{3}{*}{ El Mirador 2} & Hojas & $4.0 \pm 0.4$ & $46.5 \pm 0.1^{\text {a }}$ & $1.8 \pm 0.2$ & $3665.2 \pm 332.5$ & $1670.9 \pm 152.3$ & $3.7 \pm 0.3$ & $1.7 \pm 0.2$ \\
\hline & Ramas & $7.7 \pm 0.7$ & $47.2 \pm 0.0^{\mathrm{a}}$ & $3.6 \pm 0.3$ & $7035.8 \pm 638.2$ & $3323.0 \pm 296.7$ & $7.0 \pm 0.6$ & $3.3 \pm 0.3$ \\
\hline & Fuste & $29.3 \pm 2.8$ & $48.6 \pm 0.3^{a}$ & $14.3 \pm 1.3$ & $26906.2 \pm 2543.3$ & $13081.8 \pm 1225.3$ & $26.9 \pm 2.5$ & $13.1 \pm 1.2$ \\
\hline Total/promedio & & $41.0 \pm 1.8$ & $47.1 \pm 0.1$ & $19.7 \pm 0.9$ & $37607.2 \pm 1690.4$ & $18075.8 \pm 828.5$ & $37.6 \pm 1.7$ & $18.1 \pm 0.8$ \\
\hline \multirow[t]{3}{*}{ Cerritos 2} & Hojas & $3.4 \pm 0.5$ & $46.4 \pm 0.0^{\mathrm{a}}$ & $1.6 \pm 0.2$ & $3150.4 \pm 465.2$ & $1462.7 \pm 216.0$ & $3.2 \pm 0.5$ & $1.5 \pm 0.2$ \\
\hline & Ramas & $6.6 \pm 1.0$ & $46.2 \pm 0.2^{\mathrm{a}}$ & $3.0 \pm 0.4$ & $6047.7 \pm 893.0$ & $2794.6 \pm 412.7$ & $6.0 \pm 0.9$ & $2.8 \pm 0.4$ \\
\hline & Fuste & $24.2 \pm 4.5$ & $48.6 \pm 0.0^{\mathrm{a}}$ & $11.8 \pm 2.2$ & $22263.7 \pm 4096.1$ & $10811.3 \pm 1989.1$ & $22.3 \pm 4.1$ & $10.8 \pm 2.0$ \\
\hline Total/promedio & & $34.3 \pm 14.8$ & $47.1 \pm 0.1$ & $16.4 \pm 7.1$ & $31461.8 \pm 13554.8$ & $15068.6 \pm 6546.9$ & $31.5 \pm 13.6$ & $15.1 \pm 6.5$ \\
\hline \multirow[t]{3}{*}{ Cerritos 1} & Hojas & $1.9 \pm 0.4$ & $45.8 \pm 0.0^{\mathrm{a}}$ & $0.9 \pm 0.2$ & $1714.2 \pm 351.0$ & $785.1 \pm 160.0$ & $1.7 \pm 0.4$ & $0.8 \pm 0.2$ \\
\hline & Ramas & $3.6 \pm 0.7$ & $46.5 \pm 0.0^{\mathrm{a}}$ & $1.7 \pm 0.3$ & $3290.7 \pm 673.9$ & $1529.5 \pm 318.3$ & $3.3 \pm 0.7$ & $1.5 \pm 0.3$ \\
\hline & Fuste & $11.2 \pm 3.5$ & $48.1 \pm 0.1^{\mathrm{a}}$ & $5.4 \pm 1.7$ & $10253.5 \pm 3233.7$ & $4934.0 \pm 1572.2$ & $10.3 \pm 3.2$ & $4.9 \pm 1.6$ \\
\hline Total/promedio & & $16.6 \pm 12.9$ & $48.6 \pm 0.0$ & $7.9 \pm 6.2$ & $15258.4 \pm 11871.1$ & $7248.6 \pm 1702.4$ & $15.3 \pm 11.9$ & $7.2 \pm 5.7$ \\
\hline
\end{tabular}

B: biomasa. C: carbono.

Valores de medias ( \pm error estándar) en cada columna sin letras son estadísticamente diferentes $(p \leq 0.05)$. 


\section{Carbono acumulado por sistema}

El principal reservorio de $\mathrm{C}$ en las tres PCT fue el suelo $\left(\mathrm{F}_{6,2}=12.7, \mathrm{p}=0.0006\right)($ p. ej. PCTM2 $=65.7 \%$, PCTC2 $=53.9 \%$ y PCTC1= $71.4 \%$ y el de menor aportación el mantillo $\left(\mathrm{F}_{6,2}=113.9, \mathrm{p} \leq 0.0001\right)$ (p. ej. PCTM2= $5.3 \%$, РCTC2=7.9 \% у PCTC1= $6.9 \%)$. La PCTM2 reportó mayor acumulación de $\mathrm{C}$ en toda su estructura que PCTC2 y C1 (36.7 y $46.6 \%$ ), esto fue sustentado por la diferenciación de biomasa entre las plantaciones ligado a las propiedades del suelo. El C acumulado en la BTA $\left(\mathrm{F}_{6,2}=238.9, \mathrm{p} \leq 0.0001\right)$ de la PCTM2 fue $16.6 \%$ $(p \leq 0.0001)$ superior que la PCTC2 y más del doble que la PCTC1 (60.2\%); al igual que el mantillo (6.1 y $30.3 \%$ ) y suelo (48.0 y 41.9 ) (figura 2). El contraste del $\mathrm{C}$ acumulado entre la plantación de mayor y menor rendimiento fue de $29.0 \mathrm{Mg} \mathrm{ha}^{-1}$. Finalmente, el orden prioritario según aportes de C entre los componentes de las PCT sugiere: suelo > BTA > mantillo.

\section{Composición lignocelulósica de la madera de teca}

Las diferencias en crecimiento entre las plantaciones no supusieron cambios estadísticamente significativos en los contenidos lignocelulares entre la PCTM2 y C2, pero sí con respecto a la PCTC1 (excepto extraíbles y cenizas). A pesar de ello, los arboles de la PCTC1 presentaron alrededor del 1 y $2 \%$ más de holocelulosas y hemicelulosas $\left(\mathrm{F}_{6,2}=\right.$ 112.5, $\mathrm{p}=0.03$ y $_{6,2}=8.3, \mathrm{p}=0.001$ ) que la PCTM2 y $\mathrm{C} 2$; entre el 0.2 y $1 \%$ (aproximadamente) de celulosa $\left(F_{6,2}=14.5, p=0.03\right)$, ligninas $F_{6,2}=14.5$, $\mathrm{p}=0.07$ y extraíbles $\left(\mathrm{F}_{6,2}=0.5, \mathrm{p}=0.1\right)$ en la PCTM2 (tabla 5).

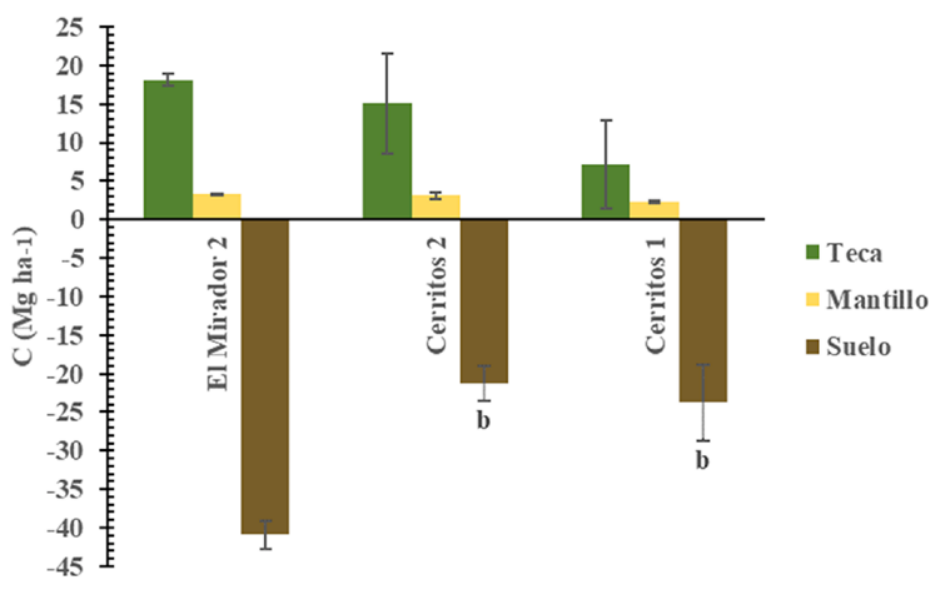

Figura 2. Acumulación de $C$ en plantaciones de teca a los 6 años en el estado de Nayarit, México. Valores de medias ( \pm error estándar) en cada barra sin letras son estadísticamente diferentes ( $\mathrm{p} \leq 0.05$ ); $\mathrm{n}=75$ (teca). $\mathrm{n}=6$ (mantillo y suelo).

Tabla 5. Composición lignocelulósica de la madera de teca a los 6 años en el estado de Nayarit, México ( $n=6)$.

\begin{tabular}{lcccccc}
\hline \multicolumn{1}{c}{ Plantación } & $\begin{array}{c}\text { Holocelulosas } \\
\mathbf{( \% )}\end{array}$ & $\begin{array}{c}\text { Celulosa } \\
\mathbf{( \% )}\end{array}$ & $\begin{array}{c}\text { Hemicelulosas } \\
\mathbf{( \% )}\end{array}$ & $\begin{array}{c}\text { Ligninas } \\
(\mathbf{\%})\end{array}$ & $\begin{array}{c}\text { Extraíbles } \\
(\mathbf{\%})\end{array}$ & $\begin{array}{c}\text { Cenizas } \\
(\mathbf{\%})\end{array}$ \\
\hline El Mirador 2 & $56.1 \pm 1.4^{\mathrm{b}}$ & $44.7 \pm 0.1^{\mathrm{a}}$ & $11.4 \pm 0.2^{\mathrm{b}}$ & $33.0 \pm 0.0$ & $9.9 \pm 0.2^{\mathrm{a}}$ & $1.1 \pm 0.0^{\mathrm{a}}$ \\
Cerritos 2 & $56.1 \pm 0.6^{\mathrm{b}}$ & $44.4 \pm 0.0^{\mathrm{a}}$ & $11.7 \pm 0.0^{\mathrm{b}}$ & $32.8 \pm 0.1^{\mathrm{b}}$ & $9.9 \pm 0.0^{\mathrm{a}}$ & $1.1 \pm 0.0^{\mathrm{a}}$ \\
Cerritos 1 & $57.1 \pm 1.1$ & $43.3 \pm 0.0$ & $13.8 \pm 1.0$ & $32.0 \pm 0.3^{\mathrm{b}}$ & $9.8 \pm 0.0^{\mathrm{a}}$ & $1.1 \pm 0.0^{\mathrm{a}}$ \\
\hline
\end{tabular}

Valores de medias ( \pm error estándar) sin letras en cada columna son estadísticamente diferentes $(p \leq 0.05)$. 


\section{DISCUSIÓN}

\section{Crecimiento de la teca}

Fue evidente que las plantaciones evaluadas tuvieron diferencias en crecimiento. En comparación con otras plantaciones de igual edad establecidas en suelos con $\mathrm{pH}=4.4$, el valor de las variables dendrométricas reportadas en China $(\mathrm{H}=5.8 \mathrm{~m}$, $\mathrm{DAP}=6.5 \mathrm{~cm})$ (Zhou et al., 2017) fueron similares al promedio encontrado en la PCTC1. Según la calidad de los sitios en Guatemala, los árboles pueden alcanzar una altura de 11 a $17 \mathrm{~m}$ (calidad baja y alta) y $A B \leq 18 \mathrm{~m}^{2}$.ha-1 (Mollinedo et al., 2016) siendo superior a lo evidenciado en las PCTM2 y C2. Basados en los rendimientos de la teca en México, la Conafor (2013a) reportó que a los 6 años puede crecer en $\mathrm{H}=6 \mathrm{~m}$, $\mathrm{DAP}=11.8 \mathrm{~cm}$ y $\mathrm{AB}=12.4$ $\mathrm{m}^{2} \mathrm{ha}^{-1}$, siendo el intermedio entre las plantaciones antes mencionadas. Cuando el suelo presenta un $\mathrm{pH}<5$ y $\mathrm{Da} \geq 1$ g. $\mathrm{cm}^{-3}$ y las condiciones hidroclimáticas del sitio no son favorables, afectan significativamente el crecimiento de la teca (Derwisch et al., 2009; Zhou et al., 2012; De Camino y Morales, 2013; Nanko et al., 2014; Chaturvedi y Raghubanshi, 2015; Zhou et al., 2017).

\section{Modelos alométricos, biomasa y carbono en plantaciones de teca}

Los modelos alométricos para la estimación de la biomasa construidos en esta investigación, tuvieron mejor ajuste que los referenciados por Kraenzel et al. (2003) $\left(R^{2}=0.98\right)$ en Panamá; Pérez y Kanninen (2003) $\left(R^{2}=0.97\right)$ y Segura y Andrade (2008) $\left(R^{2}=0.96\right.$ a 0.98$)$ en Costa Rica para BTA; Jha (2015) en hojas $\left(R^{2}=0.46\right)$, ramas $\left(R^{2}=0.59\right)$, fuste $\left(R^{2}=0.74\right)$ y BTA $\left(R^{2}=0.95\right)$; además de Chaturvedi y Raghubanshi (2015) en BTA $\left(R^{2}=0.97\right)$ en la India. Los modelos alométricos construidos a partir de parámetros biométricos como $\mathrm{H}$, DAP y la edad (variable independiente) son importantes para la estimación de la biomasa y $\mathrm{C}$ en especies vegetales (Jha, 2015). Si bien las mediciones directas de la biomasa generan modelos con mejor ajuste que los producidos por cálculos indirectos (Chaturvedi y Raghubanshi, 2015), están relacionados con los valores de ajustes obtenidos en el presente estudio.

La concentración de $\mathrm{C}$ en la biomasa vegetal de la PCTM2, C2 y C1 estuvo dentro de los parámetros sugeridos por otros autores (entre 45 y $50 \%$ ) y puede variar por efectos del método usado para su determinación (Acosta-Mireles et al., 2009; Sreejesh et al., 2013; Takahashi et al., 2012; Moya y Tenorio, 2013). El C concentrado en los suelos de las plantaciones muestreadas fue inferior a lo cuantificado en la India (1.39 a $1.64 \%$ ) (Chaturvedi y Raghubanshi, 2015), Guatemala (5\%) (Rodas, 2006) y Brasil (alrededor del $3 \%$ ) (Almeida et al., 2010). Por consiguiente, la concentración C en dicho componente pueden aumentar o disminuir por efectos de las condiciones hidroclimáticas, tipo de biomasa vegetal (especies de lento y rápido crecimiento), Da y profundidad (FAO, 2017).

Los rendimientos en BTA por hectárea entre la plantación de mayor y menor crecimiento del presente estudio difirieron el $59.4 \%$. Comparando los resultados de la PCTM2 con otros estudios, fueron superiores que los estimados en la India (31.6 Mg.ha-1) en plantaciones de 5 años (Jha, 2015) pero difieren notablemente al relacionar otra plantación de igual edad que la anterior, pero en otra región de la India (107.5 Mg.ha-1) (Sreejesh et al., 2013) y los reportados en México (43.4 Mg.ha ${ }^{-1}$ ) en plantaciones de 6 años (Vincent, 2018). Estas diferencias fueron sustentadas por la disimilitud de manejo silvícola entre las plantaciones contrastadas. Al igual que la biomasa, el $\mathrm{C}$ acumulado en la PCTM2 distó de sus competidoras pese a ello, fue mayor a lo estimado

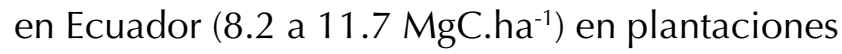
de 8 años (Jiménez y Landeta, 2009), México (8.4

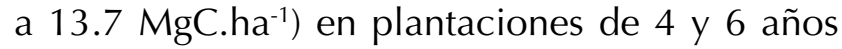
(Paradas et al., 2010; Vincent, 2018), pero inferiores según reportes de Brasil, Tailandia y la India 


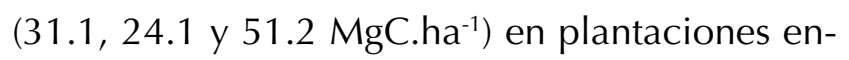
tre 5 y 6 años (Almeida et al., 2010; Masamichi et al., 2012; Sreejesh et al., 2013). La distribución del C en la BTA en este trabajo coincidió con estudios en 5 a $9 \%$ hojas, 15 a $38 \%$ ramas, 57 a $76 \%$ en fuste (Parada et al., 2010; Sreejesh et al., 2013; González-Martínez et al., 2014). El suelo fue un componente importante en almacenaje de $C$ en las tres plantaciones estudiadas, aunque dichos valores distaron de lo estimado en Panamá (225.0 MgC.ha-1) en plantaciones de 20 años (Kraenzel et al., 2003), Costa Rica (95.0 MgC.ha ${ }^{-1}$ ) a los 10 años (Ibrahim et al., 2007) y Brasil (269.0 MgC.ha-1) en 5.5 años (Almeida et al., 2010). Cambios sustanciales en la acumulación de $C$ en el suelo radica en los efectos de la $\mathrm{Da}$, profundidad del muestreo, grado de perturbación y edad de las plantaciones (Kraenzel et al., 2003; Almeida et al., 2010). En este trabajo la PCTM2 acumuló mayor C en mantillo, aunque al comparar estos rendimientos con otros trabajos fue superior a lo citado en Brasil y Panamá (2.7 y 3.2 MgC.ha-1) en plantaciones de 5.5 y 20 años (Kraenzel et al., 2003; Almeida et al., 2010). La época del muestreo y edad de la biomasa modifican la retención de $\mathrm{C}$ por dicho componente en los sistemas forestales (Schlegel, 2001; Kumar et al., 2011; Takahashi et al., 2012).

\section{Distribución lignocelulósica de la madera en teca}

El presente estudio demostró que la distribución lignocelulósica en la madera de teca no sufrió variaciones significativas observando la divergencia en crecimiento, biomasa y $\mathrm{C}$ entre PCTM2, C2 y C1. La distribución lignocelular de la madera coincidió con otros estudios. Aproximadamente, el $57 \%$ corresponde a holocelulosa, entre 33 a $47 \%$ celulosa, 13 a $35 \%$ ligninas, hasta un $15 \%$ de extraíbles y menos del $3 \%$ de cenizas (Pettersen, 1984; Windeisen et al., 2003; Moya et al., 2010; Moya y Tenorio, 2013). Los datos reportados en Brasil proveniente de plantaciones de 13 años coinciden con los del presente trabajo en $57.9 \%$ de holocelulosa, $32.5 \%$ de lignina, $8.9 \%$ de extraíbles y $0.6 \%$ de cenizas (Blanco, 2012).

\section{CONCLUSIONES}

Partiendo del manejo silvícola uniforme de las PCT estudiadas, los valores de crecimiento obtenidos en el Mirador 2 están dentro del rango reportado en plantaciones de teca establecidas en Latinoamérica. Además, coincidieron con el mejor suelo en $\mathrm{pH}, \mathrm{C}$ y $\mathrm{MO} \%$. Los modelos alométricos construidos para la estimación de la biomasa (hojas, ramas, fuste y BTA) en esta investigación, tuvieron mejores ajustes que los reportados en otros estudios; por ende, pueden ser utilizados en plantaciones de teca establecidas bajo similares condiciones hidroclimatológicas y edáficas. Las diferencias en biomasa y $\mathrm{C}$ acumulado la PCTM2 y C1 fue alrededor del $50 \%$, además, están por debajo de lo cuantificado en algunos estudios en plantaciones de mayor y menor edad.

El suelo fue el componente que más $\mathrm{C}$ almacenó en las tres plantaciones evaluadas (53 hasta el $71 \%)$; sin embargo, los valores encontrados en la plantación El Mirador 2 distan del promedio referenciado en otras investigaciones. El mantillo fue el componente que menor $\mathrm{C}$ retuvo en relación con la biomasa arbórea y suelo, aunque los datos obtenidos en la plantación de mejor rendimiento son de mayor trascendencia que en plantaciones de Brasil y Panamá. Cambios en el crecimiento de la teca no son factores significativamente determinantes en la composición lignocelulósica de su madera. Se recomienda en futuros estudios de biomasa y $\mathrm{C}$ en plantaciones comerciales sea muestreada la biomasa subterránea, además, de los nutrientes en suelo puesto que, generaran mayor información sobre los parámetros que condicionan la eficiencia de la teca en torno a su aportación al cambio climático. 


\section{AGRADECIMIENTOS}

Los autores agradecemos al licenciado Álvaro Navarro Esquivel, propietario de la empresa Agroforestal Nayarita S. A., y colaboradores por permitir la ejecución del proyecto sus plantaciones, al Departamento de Producción Forestal por el apoyo financiero a través del proyecto n. ${ }^{\circ} \mathrm{P} 3 \mathrm{E}: 224301$ 2327924, al Departamento de Madera, Celulosa y Papel (DMCyP) por permitir la realización de la maestría al autor principal, al Consejo Nacional de Ciencia y Tecnología (Conacyt) por financiar los estudios de maestría al autor principal, a la doctora Yareni Perroni Ventura (Universidad Veracruzana) y Lovren A.R.G.h., por apoyar indirectamente el proyecto.

\section{CONFLICTO DE INTERESES}

Los autores declaran no tener conflicto de intereses.

\section{CONTRIBUCIÓN POR AUTOR}

B.A. R-B., E. H-A. y E. S-P. establecieron el contacto con el propietario de la plantación, redactaron la propuesta de investigación, realizaron los muestreos en campo y experimentación en el laboratorio, construyeron los modelos alométricos, redactaron los resultados y escribieron el primer borrador del artículo. R. R-M. analizó los resultados para el componente suelo, contribuyó en la discusión y revisión general del documento. A.G-R. aportó en la construcción de los modelos alométricos, realizó el mapa, hizo el análisis estadístico y revisó el documento final. E.V-V. y R.S-H. suministraron las referencias para hacer los muestreos en campo para el componente suelo, analizaron sus propiedades y almacenamiento de carbono, apoyaron en la redacción e interpretación de los resultados y búsqueda de referencias. Si embargo, todos los autores ayudaron en la construcción y elaboración del artículo lo cual, son los directos responsables.

\section{REFERENCIAS}

Acosta-Mireles, M., Carrillo-Anzures, F. y Díaz, L. (2009). Determinación del carbono total en bosques mixtos de Pinus patula Schl. et Cham. Terra Latinoamericana, 27(2),105-114.

https://doi.org/10.21829/myb.2007.1311233

Almeida, E., Júnior, J. y Finger, Z. (2010). Determinação do estoque de carbono em teca (Tectona grandis L.F.) em diferentes idades. Ciência Florestal, 20(4), 559-568.

http://doi.org/10.5902/198050982414

Blanco, F. (2012). Caracterização tecnológica da madeira jovem de teca (Tectona grandis L.f.) (Dissertação Mestrado em Ciência e Tecnologia da Madeira). Universidade Federal de Lavras. Lavras, Mina Gerais, Brasil.

https://doi.org/10.12953/2177-6830/rcm.v8n2p54-63

Cancino, J. (2006). Dendrometría básica. Universidad de Concepción, Chile.

Chaturvedi, R. y Raghubanshi, A. (2015). Assessment of carbon density and accumulation in mono-and multi-specific stands in Teak and Sal forests of a tropical dry region in India. Forest Ecology and Management, 339, 11-21.

https://doi.org/10.1016/j.foreco.2014.12.002

Comisión Nacional Forestal (Conafor) (2013). Programa de desarrollo de plantaciones forestales comerciales, a 15 años de su creación. México D. F.: Conafor.

Comisión Nacional Forestal (Conafor) (2013a). Evaluación de la situación actual de proyectos de plantaciones forestales comerciales finiquitados. México D. F.: Conafor.

De Camino, R. y Morales, J. (2013). Las plantaciones de teca en América Latina: mitos y realidades. Serie Técnica. Centro Agronómico Tropical de Investigación y Enseñanza (Catie) División de Investigación y Desarrollo. Turrialba, Costa Rica.

Derwisch, S., Schwendenmann, L., Olschewski, R. y Hölscher, D. (2009). Estimation and economic evaluation of aboveground carbon storage of Tectona grandis plantations in Western Panama. New Forests, 37(3), 227-240.

https://doi.org/10.1007/s11056-008-9119-2 
Diario Oficial de la Federación (DOF) (2002). Norma Oficial Mexicana NOM-021-RECNAT-2000, que establece las especificaciones de fertilidad, salinidad y clasificación de suelos. Estudios, muestreo y análisis. Diario oficial de la federación. México.

https://doi.org/10.22201/iiec.20078951e.1996.106. 29193

Food and Agriculture Organization of the United Nations (FAO) (2017). Soil Organic Carbon: the hidden potential. Roma: FAO.

Germplasm Resources Information Network (GRIN) (2018). National Plant Germplasm System: Taxonomy for plants resources. Information network.

https://doi.org/10.21273/hortsci.43.5.1365

González, S. y Gómez, G. (2015). Efecto invernadero y cambio climático: antecedentes, situación actual y perspectivas. Repositorio abierto de la Universidad de Cantabria, España.

González-Martínez, Á., Rojas-Hernández, J., Jiménez-Gómez, R. y Chavarría-Ñamendi, F. (2014). Evaluación del crecimiento, potencial de secuestro y fijación de carbono de dos especies forestales en el Sistema Agroforestal Taungya en Rivas, Nicaragua. Revista Forestal Mesoamerica Kurú, 11(26), 12-18.

https://doi.org/10.18845/rfmk.v11i26.1590

González-Molina L., Etchevers-Barray, J. y Hidalgo-Moreno, C. (2008). Carbono en suelos de ladera: factores que deben considerarse para determinar su cambio en el tiempo. Agrociencia, 42, 741-751.

Grünewald, F., Chapuli, E. y Palanca, A. (2014). Producción industrial de biomasa de microalgas. Una salida energética a la crisis alimentaria, energética y medioambiental. Madrid: Instituto tecnológico del plástico (Aimplas).

García, E. (1987). Modificaciones al sistema de clasificación climática de Köppen (para adaptarlo a las condiciones de la República Mexicana). México D.F.: Offset Larios.

Ibrahim, M., Chacón, M., Cuartas, C., Naranjo, J., Ponce, G., Vega, P., Casasola, F. y Rojas, J. (2007). Almacenamiento de carbono en el suelo y la biomasa arbórea en sistemas de usos de la tierra en paisajes ganaderos de Colombia, Costa Rica y Nicaragua. Agroforestería en las Américas, 45, 27-36.

https://doi.org/10.18845/rfmk.v9i22.360
Instituto Nacional de Estadística y Geografía (Inegi) (2017). Anuario estadístico y geográfico de Nayarit. México D. F.: Inegi.

Intergovernmental Panel on Climate Change (IPCC) (2007). Climate Change 2007: The Scientific Basis Contribution of Working Group I to the Fourth Assessment Report of the Intergovernmental Panel on Climate Change. En R. Alley, T. Berntsen, N. Bindoff, Z. Chen, A. Chidthaisong, P. Friedlingstein y J. Gregory. This Summary for Policymakers was formally approved at the 10th Session of Working Group I of the IPCC (p 18), París.

https://doi.org/10.1017/cbo9781107415324.004

Jha, K. (2015). Carbon storage and sequestration rate assessment and allometric model development in young teak plantations of tropical moist deciduous forest, India. Journal Forestry Research, 26, 589-604.

https://doi.org/10.1007/s11676-015-0053-9

Jiménez, E. y Landeta, A. (2009). Producción de biomasa y fijación de carbono en plantaciones de teca (Tectona grandis Linn F). Campus Prosperina - ESPOL. Guayaquil, EC, Facultada de Ingeniería Mecánica y Ciencia de la Producción. Escuela Superior Politécnica del Litoral Campus Gustavo Galindo, Ecuador.

https://doi.org/10.21931/rb/2018.03.02.7

Kraenzel, M., Castillo, A., Moore, T. y Potvin, C. (2003). Carbon storage of harvest-age teak (Tectona grandis) plantations, Panama. Forest Ecology and Management, 173, 213-225.

https://doi.org/10.1016/S0378-1127(02)00002-6

Kumar, J., Sajish, P., Kumar, R. y Patel, K. (2011). Biomass and net primary productivity in three different aged Butea forest ecosystems in Western India, Rajasthan. Our Nature, 9(1), 73-82.

https://doi.org/10.3126/on.v9i1.5736

Masamichi, T., Dokrak, M., Samreong, P. y Keizo, H. (2012). Carbon cycling in teak plantations in comparison with seasonally dry tropical forests in Thailand. En A. B. Juan (ed), Forest ecosystems-more than just trees (pp 209-229).

https://doi.org/10.5772/30196 
Mollinedo, M., Herrera, M. y Muñoz, F. (2016). Caracterización del crecimiento de plantaciones jóvenes de teca (Tectona grandis Linn f.) y estimación de curvas de índice de sitio en el área septentrional de la República de Guatemala. Madera y Bosques, 22(2), 89-103.

https://doi.org/10.21829/myb.2016.2221327

Moya, R. y Tenorio, M. (2013). Características de combustibilidad de diez especies de plantaciones de rápido crecimiento en Costa Rica. Revista Forestal Mesoamericana Kurú, 10(24), 26-33.

https://doi.org/10.18845/rfmk.v10i24.1321

Moya, R., Muñoz, A. y Berrocal, J. (2010). Teca: Tectona grandis L.f. Verbenaceae. Ficha técnica 7. In Tecnología de madera de plantaciones forestales: Fichas técnicas. Revista Forestal Mesoamericana Kurú, 7, 121-135.

Nanko, K., Ugawa, S., Hashimoto, S., Imaya, A., Kobayashi, M., Sakai, H., Ishizuka, S., Miura, S., Tanaka, N., Takahashi, M. y Kaneko, S. (2014). A pedotransfer function for estimating bulk density of forest soil in Japan affected by volcanic ash. Geoderma, 213, 36-45.

https://doi.org/10.1016/j.geoderma.2013.07.025

Núñez, C. (2008). Análisis químico de los recursos fibrosos para pulpa. Universitaria en Celulosa y Papel. Ingeniería Química. Facultad de Ciencias Exactas. Universidad Nacional de Misiones. Misiones, Argentina.

https://doi.org/10.20937/rica.2017.33.02.12

Ordóñez, J. y Masera, O. (2001). La captura de carbono ante el cambio climático. Madera y Bosques, $7(1), 3-12$.

https://doi.org/10.21829/myb.2001.711314

Parada, S., Gallegos, A., Velázquez, M. y Hernández, E. (2010). Guía para la medición de biomasa en plantaciones forestales de teca (Tectona grandis L.f.) y cedro (Cedrela odorata L.) para determinar su potencial de captura de carbono. CUCBA. Departamento Forestal. Universidad de Guadalajara, México.

https://doi.org/10.5154/r.rchscfa.2011.09.070

Parra, A., Insuasti, A., Landazury, B. y Preciado, B. (2013). Evaluación de gases de efecto invernadero (GEI) en sistemas ganaderos asociados con pasto kikuyo (Pennisetum clandestinum Hoechst Ex Chiov). Revista Colombiana de Ciencia Animal, 6(1), 36-43.

Pérez, L. y Kanninen, M. (2003). Aboveground biomass of Tectona grandis plantations in Costa Rica. Journal of Tropical Forest Science, 15(1), 199-213.

Pettersen, R. (1984). The chemical composition of wood. En R. M. Rowell (ed.), The chemistry of solid wood (pp. 57-126). Washington D.C.: ACS Advances in Chemistry Series No. 207, American Chemical Society.

https://doi.org/10.1021/ba-1984-0207.ch002

Rodas, C. A. (2006). Efecto del establecimiento de plantaciones forestales de teca (Tectona grandis) en áreas de potrero sobre las características del suelo en Petén, Guatemala (tesis Magister en Ciencia de Agroforestería Tropical). CATIE. Turrialba, Costa Rica.

https://doi.org/10.18779/cyt.v3i1.135

Segura, M. y Andrade, H. (2008). ¿Cómo hacerlo? ¿Cómo construir modelos alométricos de volumen, biomasa o carbono de especies leñosas perennes? Agroforestería de las Américas, 46, 89-96.

SAS Institute Inc. (2009). SAS/STAT 9.0 User's Guide Second Edition. Raleigh, NC, EE.UU.: SAS Institutelnc. s/p.

https://doi.org/10.1787/9789264056251-18-en

Schlegel, B., Gayoso, J. y Guerra, J. (2001). Manual de procedimientos de muestreo de biomasa forestal. Medición de la capacidad de captura de carbono en los bosques de Chile y promoción en el mercado mundial. Proyecto Fondef. Universidad Austral de Chile, Valdivia, Chile.

https://doi.org/10.4067/s0717-92002005000200005

Schlegel, B. (2001). Estimación de biomasa y carbono en bosques del tipo forestal siempre verde. Simposio Internacional Medición y Monitoreo de la Captura de Carbono en Ecosistemas Forestales. Valdivia, Chile, Universidad Austral de Chile.

https://doi.org/10.4067/s0717-92002005000200005

Sreejesh, K., Thomas, T., Rugmini, P., Prasanth, K. y Kripa, P. (2013). Carbon sequestration Potential of Teak (Tectona grandis) plantations in Kerala. Research Journal of Recent Sciences, 2, 167-170. 
Takahashi, M., Marod, D., Panuthai, S. y Hirai, K. (2012). Carbon cycling in teak plantations in comparison with seasonally dry tropical forests in Thailand. In Forest Ecosystems-More than Just Trees. InTech.

https://doi.org/10.5772/30196

Tappi Useful Method (1954). Celulosa Cross y Bevan (Test Method T 9M). Atlanta, EE. UU.: Technical Association of the Pulp and Paper Industry.

Tappi Useful Method (2007). Preparation of wood for chemical analysis (Test Method T 264 cm-2007). Atlanta, EE. UU.: Technical Association of the Pulp and Paper Industry.

Tappi Useful Method (2007). Solvent extractives of wood and pulp. (Test Method T $204 \mathrm{~cm}$-2007). Atlanta, EE. UU.: Technical Association of the Pulp and Paper Industry.

Tappi Useful Method (2011). Acid-insoluble lignin in wood and pulp (Test Method T 222 om-11). Atlanta, EE. UU.: Technical Association of the Pulp and Paper Industry..

Tappi Useful Method (2012). Ash in wood, pulp, paper and paperboard: combustion at $525^{\circ} \mathrm{C}$ (Test Method T 211 om-12). Atlanta, EE. UU.: Technical Association of the Pulp and Paper Industry.

Vincent, V. (2018). Estimación de biomasa y carbono en plantaciones de teca (Tectona grandis L.f.) por método indirecto y mapeo mediante sistemas de información geográfica (tesis Maestría en Ciencia de Productos Forestales). Universidad de Guadalajara, Departamento de Madera, Celulosa y Papel. Zapopan, México.

https://doi.org/10.5154/r.rchscfa.2017.07.050

Windeisen, E., Klassen, A. y Wegener, G. (2003). On the chemical characterisation of plantation teakwood from Panama. Holzals Rohund Werkstoff, 61, 416-418.

https://doi.org/10.1007/s00107-003-0425-2

Wise, I., Marphy, M. y Adieco, D. (1946). Chlorite Holocellulose, its Fractionation and Beating on Summative Wood Analysis and on Studies on the Hemicelluloses. Paper Trade, 122, 11-19.

Zhou, Z., Liang, K., Xu, D., Zhang, Y., Huang, G. y Ma, H. (2012). Effects of calcium, boron and nitrogen fertilization on the growth of teak (Tectona grandis) seedlings and chemical property of acidic soil substrate. New Forests, 43, 231-243.

https://doi.org/10.1007/s11056-011-9276-6

Zhou, Z., Liu, S., Liang, K., Ma, H. y Huang, G. (2017). Growth and mineral nutrient analysis of teak (Tectona grandis) grown on acidic soils in south China. Journal of Forestry Research, 28, 503-511.

https://doi.org/10.1007/s11676-016-0324-0

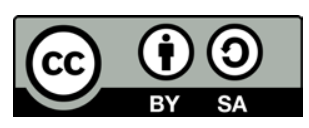

Colombia Forestal •ISSN 0120-0739 • e-ISSN 2256-201X • Bogotá-Colombia • Vol. 22 No. 2 • Julio-diciembre de 2019 • pp. 15-29 